\title{
Global Navigation Satellite System: A Review
}

\author{
Anupam Sharma ${ }^{1}$, Amit Sarkar ${ }^{2}$ \\ ${ }^{1}$ (Department of Electrical \& Electronics Engineering, Goa college of engineering, Farmagudi, Goa-403401) \\ ${ }^{2}$ (School of Electrical Sciences, Indian Institute of Technology Bhubaneswar, Odisha-752050)
}

\begin{abstract}
Recently, Radio Based Global Navigation Satellite System have become a part of our daily lives for location based services, Real time tracking \& Turn by Turn navigation. Global Navigation Satellite System is a generic term used for a constellation of satellites with worldwide coverage that function to transmit positioning and timing signals to receivers located on earth with software control over some functions. This paper tries to explain the Software Defined Radio based Global Navigation Satellite System receiver along with present \& future prospects.
\end{abstract}

Keywords: GNSS, SDR, GPS, GLONASS, GALILEO, COMPASS, QZSS, IRNSS

\section{INTRODUCTION}

"Transit" was the first ever satellite navigation system introduced by U.S. military in 1960s.

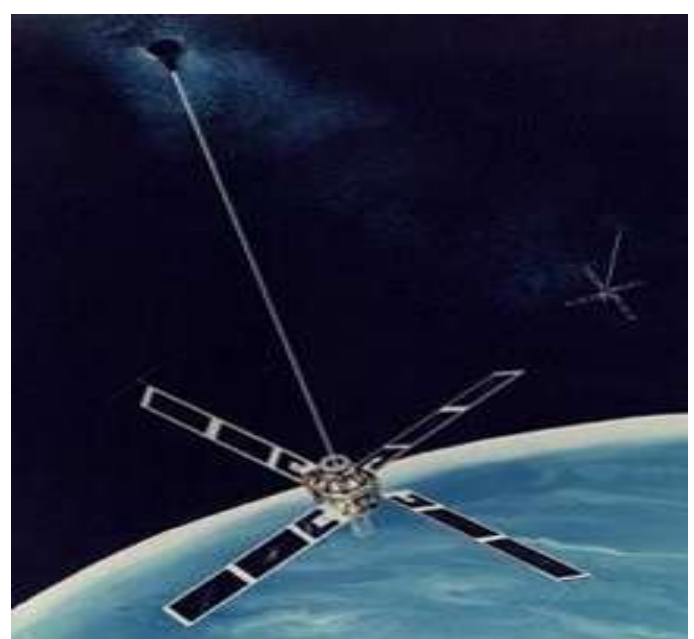

Fig.1. Transit Navigation System [1]

Transit navigation system was primarily used by the U.S. Navy to get accurate information about its ships \& submarines. In 1865, Maxwell predicted electromagnetic waves propagation. Later in 1886, Henrich hertz confirmed Maxwell theory. Hertz's experimental work demonstrated that physical objects can reflect Radio waves [2]. The presence of reflected wave can be sensed by Radar to determine the existence of a target which is the basic principle behind the working of global navigation satellite system [3]. The Angular position \& target distance can be determined from arrival direction of reflected wave-front \& time taken for the radar signal from transmitter to the receiver respectively [4].

\section{GLOBAL NAVIGATION SATELLITE SYSTEM (GNSS) RECEIVER}

A GNSS receiver is an electronic device that process the signals in space transmitted by the satellites in digital domain to determine position, velocity and time of the user [5].

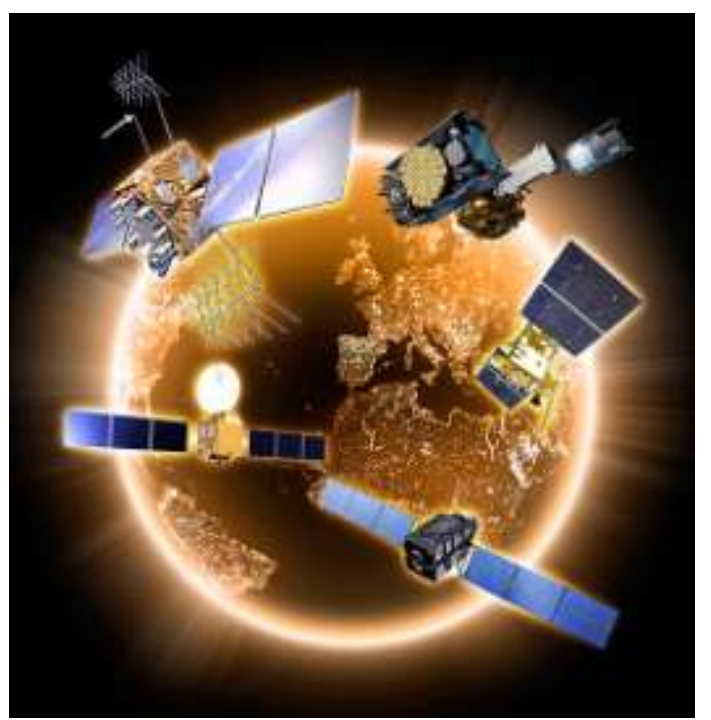

Fig.2. GNSS Satellite Constellation [6]

To multiplex several satellites signals onto the same frequency, most of the GNSS use Code Division Multiple Access technique to assign Pseudo-Random noise code to each satellite that modulates the transmitted signal. Digital modulation scheme provides more information carrying capacity, data security, better quality communication \& Radio frequency spectrum sharing as per the requirement of today's generation of wireless communication system [7]. 


\section{THE EVOLVING GNSS WORLD}

For the benefit of scientific community and society, International GNSS Service function as a key part of Global Geodetic Observing System [8]. Global Positioning System (GPS), Owned by United States Government and operated by United States Air Force has been fully functional since 1993. Currently there are 31 satellites out of the planned 32 in orbit organized at an inclination of $55^{\circ}$ to the Equator on 6 orbital planes at an altitude of 20, 180 $\mathrm{km}$ around the earth, ensuring that at least 4 satellites are in radio communication with any point on earth. Code Division Multiple Access modulation technique encode and distinguish signals from individual satellites and broadcast on $\mathrm{L}_{1}$ and $\mathrm{L}_{2}$ bands [9]. Space, Control \& User are three segments of GPS. The operational concept of GPS is based upon ranging of satellites. Each GPS satellite act as a precise reference point, transmit an accurate time and position signal [10].

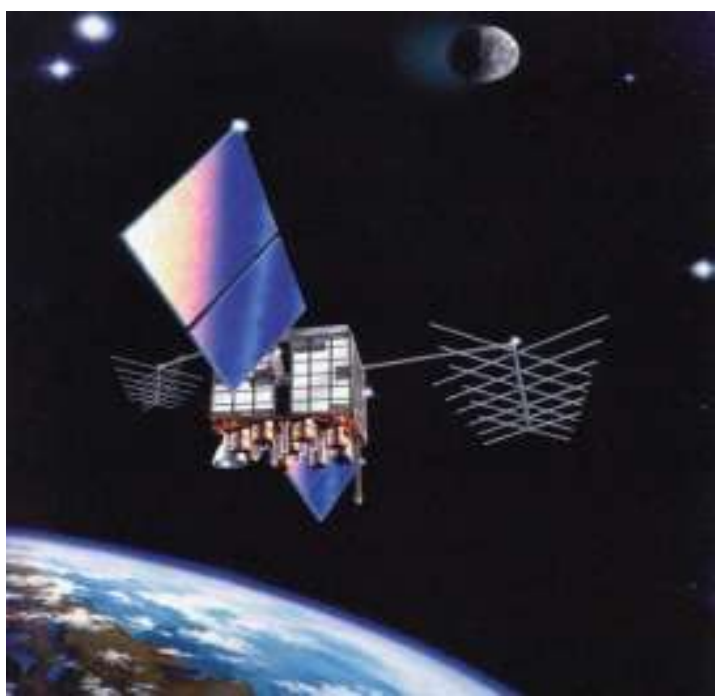

Fig.3. GPS Navigation System [11]

GLONASS, the only other fully functional \& operational GNSS, developed by Russian Federal Space Agency and maintained by Roscosmos State Corporation fully functional since 2011 [12]. Currently there are 24 satellites out of planned 27, operational from 122 satellites, in orbit located with an inclination of $48^{\circ}$ from the Equator on 3 orbital planes. 12 more satellites are being planned to be launched from March, 2017 to Q4, 2018. GLONASS has an accuracy of $20 \mathrm{~m}$ in public signal and $10 \mathrm{~m}$ in the military signal. Frequency Division Multiple Access modulation technique distinguish between satellite signals and broadcast on $\mathrm{L}_{1}$ and $\mathrm{L}_{2}$ Bands [13]. GLONASS-K2 is now in design phase and GLONASS-KM is in research phase.

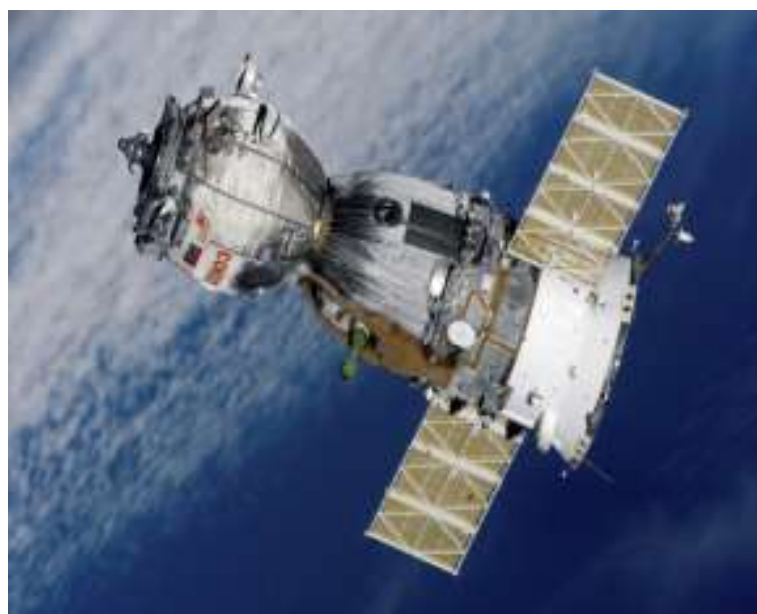

Fig.4. GLONASS Navigation System [14]

GALILEO is the GNSS currently developed by European Union [15] through European GNSS agency [16] and European Space agency [17]. Currently there are 18 satellites out of planned 30 in orbit located with an inclination of $56^{\circ}$ on 3 orbital paths around the earth, fully functional since December 2016. It utilizes Code Division Multiple Access modulation technique to differentiate between satellite signals and broadcast on $\mathrm{L}_{1}, \mathrm{E}_{5}$ and $\mathrm{E}_{6}$ Bands [18].

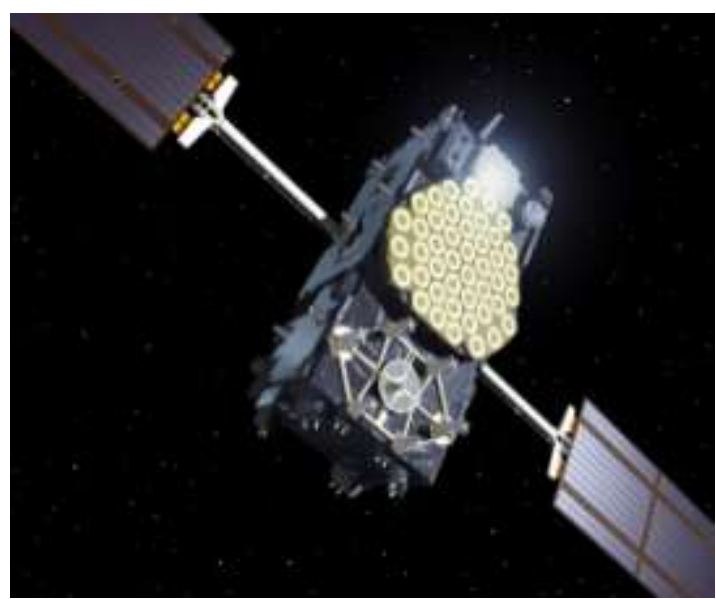

Fig.5. GALILEO Navigation System [19]

COMPASS also known as Beidou-2, is a second generation navigation system being developed by China and operated by China National Space Administration [20], fully operational in Asia Pacific region since 2012 [21]. Currently there are 21 satellites out of planned 35 in orbit. It utilizes Code Division Multiple Access modulation technique to differentiate between satellite signals and broadcast on $\mathrm{E}_{1}, \mathrm{E}_{2}, \mathrm{E}_{5} \mathrm{~B}$ and $\mathrm{E}_{6}$ Bands. China Aerospace Science and Technology Corporation are mainly responsible for the launch of satellites [22]. 


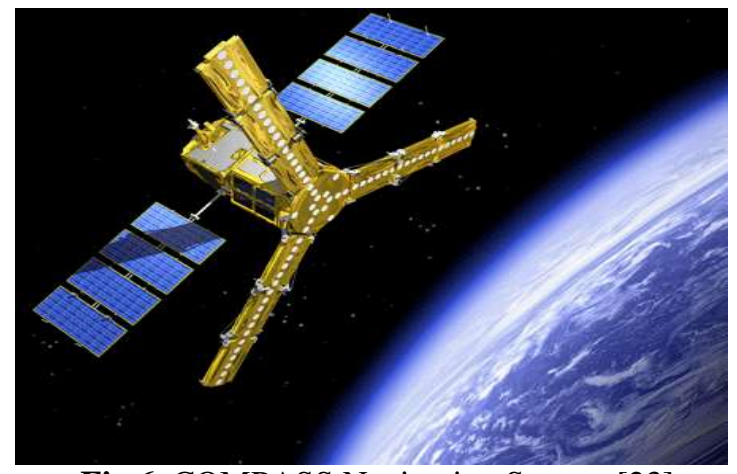

Fig.6. COMPASS Navigation System [23]

Quasi-Zenith Satellite System (QZSS), is a Satellite based augmentation system, developed in 2010 \& operated by Japan Aerospace Exploration Agency [24], mainly composed of satellites in quasizenith orbits, also known as "Japanese GPS", utilize three satellites each $120^{\circ}$ apart [25].

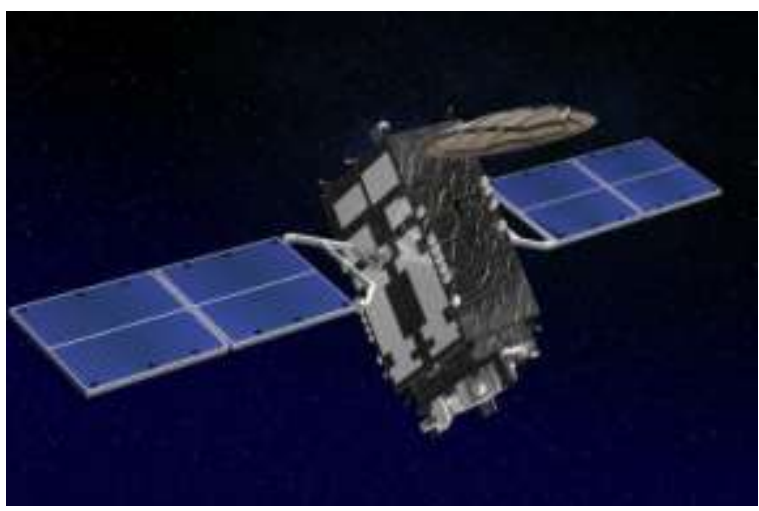

Fig.7. GNSS Augmentation QZSS [26]

Indian Regional Navigation Satellite System (IRNSS), developed in 2013 \& operated by International Space Research Organization [27]. Currently there are 6 satellites out of planned 7 in orbit, also known as Navigation with Indian Constellation [28].

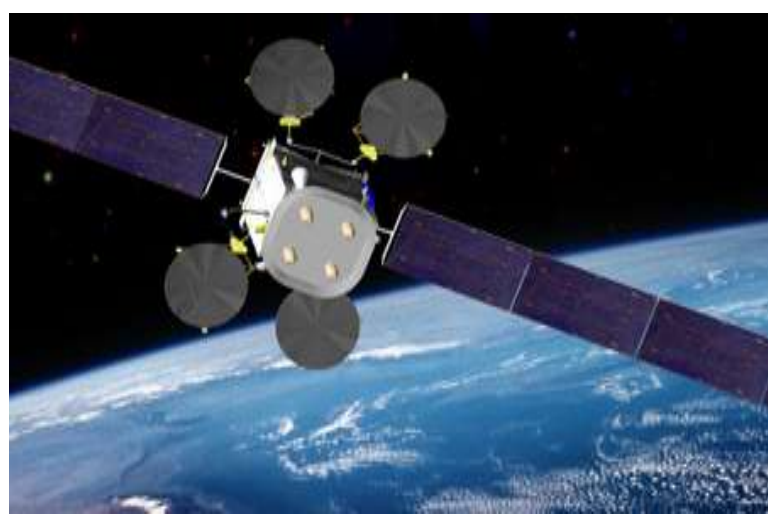

Fig.8. IRNSS [29]

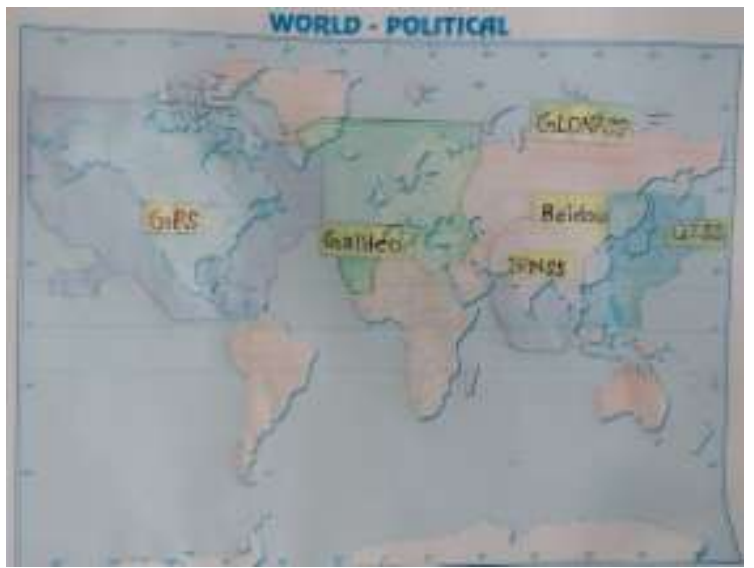

Fig.9. Evolving GNSS across the Globe

\section{SOFTWARE DEFINED RADIO (SDR) BASED GNNS RECEIVER}

Hardware GNSS Receivers are designed in a highly specialized way, generally more efficient than software GNSS receivers from the point of view of power consumption and computational load. Whereas, Software GNSS Receivers allow huge flexibility, many features of the receiver can be modified just through software, depending on the user's need \& working condition. Therefore, Software Defined Radio (SDR) based GNSS receiver is an ideal GNSS receiver [30].

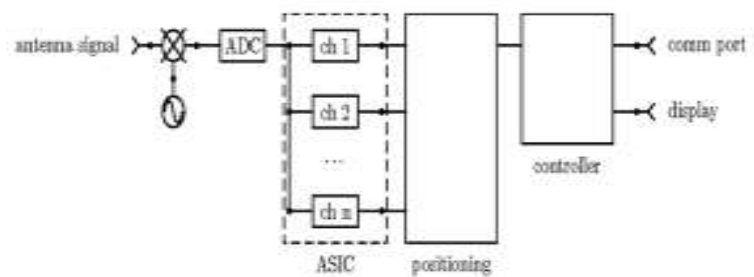

Fig.10. Block diagram of a GNSS Receiver [31]

A single Antenna will receive all the satellite signals. Receiver splits antenna signal into several identical copies after down conversion using only a single mixer (per frequency band). After down conversion, lower Intermediate Frequency signal is digitized by an Analog to Digital Converter. This digital signal can easily be reproduced to feed the distinct channels. Each channel will search for propagation delay \& navigation message of a different satellite. Signature matching process is implemented on an Application Specific Integrated Circuit due to a Doppler shift. Target motion also changes the radar's frequency that is not predictable if the receiver position is unknown.

After signature matching, demodulator decode the navigation message of that satellite \& channel is then said to be locked onto that satellite. Down conversion from Intermediate Frequency to baseband is done via a digital multiplication to 
decode navigation message in a certain channel. The precision of location determination can be increased by frequency and phase setting of the numerical oscillator. Most receivers have a communication \& display port to connect the device with a personal computer for depicting target's location [32]. All these blocks can be found in an old Rogue receiver as shown below.

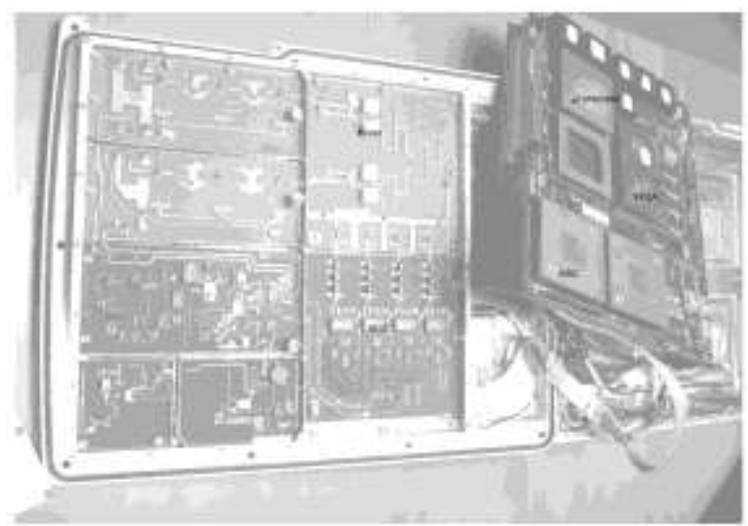

Fig.11. Photograph of an old GNSS Receiver [33]

For the effective utilization of limited spectrum resources, Software Defined Ratio (SDR) has been subject to tremendous research for the last three decades or so. With the advancement of science and engineering, SDR technology is shifting into other application domains such as cognitive radio and Dynamic spectrum access, where the waveform is completely defined in software [34].

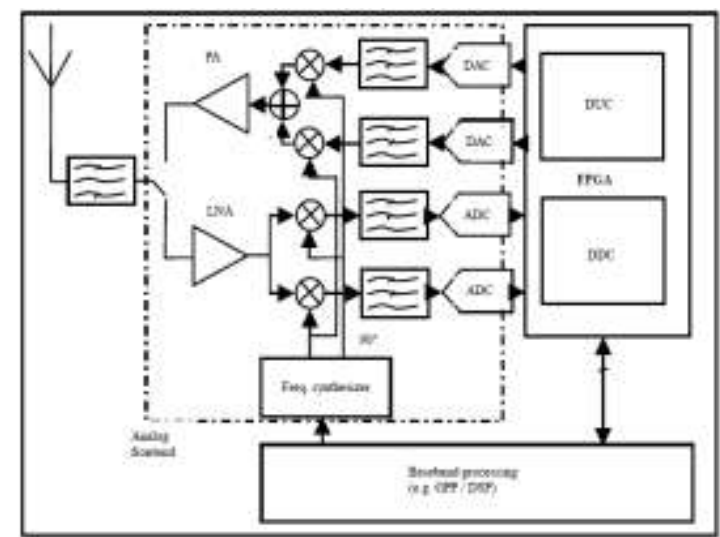

Fig.12. Common SDR Architecture [35]

The SDR system may be classified on the basis of processing capabilities, type of radio frequency front-end, cost, development software and flexibility. Some examples of SDR prototyping system available, in decreasing order of cost, are Flexcomm [36], Lyrtech [37], Typhoon from Datasoft [38], GNU Radio [39] and Ettus Research respectively [40]. Nowadays, both SDR hardware [41] and software [42] are available at a very low cost [43].

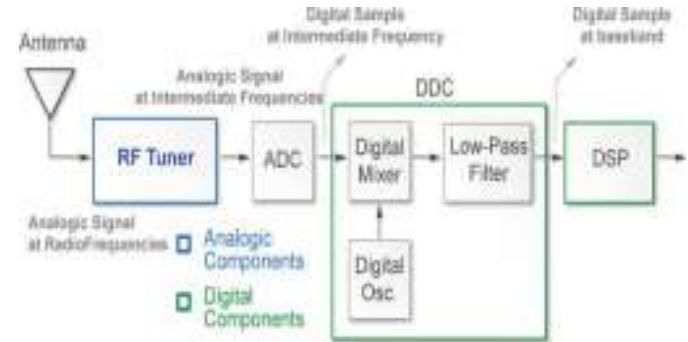

Fig.13. Block diagram of SDR Receiver [44]

At first, analog signals are converted to Intermediate Frequencies (IF) by RF tuner. Next, IF signal is fed to Analog to Digital Converter (ADC). These digital signals are then passed through Digital Down Converter (DDC), a monolithic chip, which is the key part of SDR system. DDC consist of a digital mixer, a digital local oscillator and a Finite Impulse Response (FIR) Low-Pass filter. The local oscillator $\&$ digital mixer shift the intermediate frequency digital samples to baseband, whereas FIR low pass filter limits final signal bandwidth [45].

DDC consists of adders, multiplier and shift registers for the implementation of each of its parts. To reduce the sample rate, decimation is performed, which can be as little as twice the useful signal highest frequency component [46]. Without significantly affecting the quality of results, reduction can be applied up to an extra twenty percent [47]. Finally demodulating and decoding are performed in Digital Signal Processing blocks using baseband signals.

To operate a Software Defined Radio device from an FPGA running Digital Signal Processor or from a personal computer, software is required for enabling interaction. To provide low level interface functions, a framework should be created before developing software. Since 1980, several attempts have been made as listed below:

- $\quad$ The Software radio (1980-1985) [48]

- National Instruments- LabVIEW (1986-Present) [49]

- US Military- SPEAKeasy I (1992-1995) [50]

- $\quad$ MIT SpectrumWare (1994-199) [51]

- US Military-SPEAKeasy II (1995-1997) [52]

- US Military-Joint Tactical Radio System (1997Present) [53]

- Trinity College-IRIS (1999-Present) [54]

- Vanu Software Radio (2001-Present) [55]

- $\quad$ GNU Radio (2001-Present) [56]

- $\quad$ Flex-Radio SDR-1000 (2002-Present) [57]

- Tsao, SDR Framework (2002) [58]

- Universidad de Kansas-Agile Radio (2003) [59]

- California Institute of Technology- CallRadio (2005-Present) [60]

- $\quad$ Rice- WARP (2006-Present) [61]

- High Performance SDR (2006-Present) [62] 
- Virginia Tech- Open Source SCA Implementation (2006-Present) [63]

- Lyrtech- Small Form Factor SDR (2007Present) [64]

- Virginia Tech- Cognitive Engine (2007-Present) [65]

- HYDRA (2007) [66]

- P-HAL (2008-Present) [67]

- Microsoft-SORA (2009-Present) [68]

- Karlsruhe Institute of TechnologyMATLAB/Simulink/USRP (2009) [69]

- MathWorks- MATLAB/ Simulink/ USRP (2011-Present) [70]

\section{CONCLUSION}

In the area of Global Navigation Satellite System, Software Defined Radio can act as a key technology and can provide necessary flexibility to Adaptive, Cognitive and Intelligent Radio in achieving their full potential with reduced cost and increased system efficiency. Indian Space research Organization is planning to develop SDR based GNSS receiver which will be completed by 2019 .

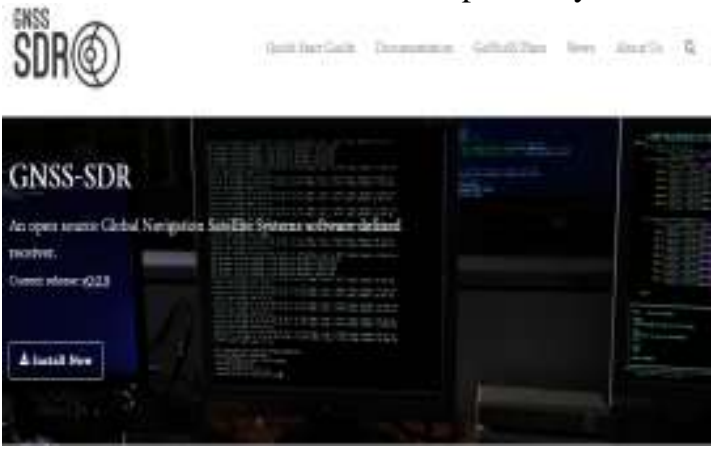

Fig.14. GNSS SDR [71]

\section{ACKNOWLEDGEMENTS}

I sincerely thanks Dr. Nitin Sharma for giving me the opportunity to write this paper.

\section{REFERENCES}

[1] www.jhuapl.edu

[2] Graham hall, Maxwell's electromagnetic theory and special relativity, Philosophical Transactions of The Royal Society A, 2008, 1849-1860.

[3] Shahram Mohanna, Detection, Identification \& Tracking of Flying Objects in three Dimensions using Multistatic Radars, International Journal of Communications, Network and system sciences, 2009, 6, 486-490.

[4] Tarig Ibrahim Osman, Radar Target Detection Simulation, International Journal of Engineering Research and Applications, 2014, 4, 2248-9622.

[5] www.navipedia.net
[6] www.gpsworld.com

[7] Manoj Barnela, Digital Modulation Schemes Employed in Wireless Communication: A Literature Review, International Journal of Wired \& Wireless communication, 2014, 2, 2319-9520.

[8] www.igs.org

[9] www.gps.gov

[10] www.navcen.uscg.gov

[11] www.afspc.af.mil

[12] en.roscosmos.ru

[13] www.glonass-iac.ru

[14] www.russianspaceweb.com

[15] europa.eu

[16] gsa.europa.eu

[17] www.esa.int

[18] www.insidegnss.com

[19] www.unoosa.org

[20] www.cnsa.gov.cn

[21] en.beidou.gov.cn

[22] www.spacechina.com

[23] www.mgex.igs.org

[24] www.jaxa.jp

[25] qzss.go.jp

[26] www.eetimes.com

[27] www.isro.gov.in

[28] www.vssc.gov.in

[29] www.bbc.com

[30] G. MacCougan \& P.L.Normark, Satellite Navigation Evolution: The Software GNSS Receiver, Inside GNSS, 2005, 1, 48-55.

[31] Gnss.be

[32] Peyton Z. Peebles, Radar Principles (John Wiley \& Sons, UK, 1998).

[33] Geodaf.mt.asi.it

[34] J. Mitola, The Software Radio, Proceedings of IEEE National Telesystem Conference, 1992, 13-15.

[35] Sverre Wichlund, Software Defined Radio Prototyping with Visual C++ express and Code Composer Studio, Wireless engineering and technology, 2012,3, 52-62.

[36] www.spectrumsignal.com

[37] www.lyrtech.com

[38] www.datasoft.com

[39] www.gnuradio.org

[40] www.ettus.com

[41] www.amazon.com

[42] www.sdrsharp.com

[43] www.github.com

[44] Jose Fernandez, Software Defined Radio: Basic Principal and Applications, Proceedings of Enero, 2015, 2357-5328.

[45] V. Giannini, Baseband analog circuits for software defined radio, (Springer, 2008).

[46] B. Sklar, Digital communication fundamental and application, (Prentice Hall, 2001). 
[47] R. H. Hosking, Software Defined Radio Handbook, (8 Edison, 2010).

[48] G.D. Space System Technology group, New Research Lab Leads to Unique Radio Receiver, E-System team Magazine, 1985, 6-7

[49] www.ni.com

[50] D. W. Upmal, Speakeasy: The Military Software Radio, IEEE Communication Magazine, 1995, 56-61

[51] Design and Implementation of Software Radios using a general purpose Processor, doctoral diss., Massachusetts Institute of Technology, Cambridge, MA, 1999.

[52] R. Vidano, Speakeasy II: an IPT approach to software programmable Radio Development, MILCOM 97 Proceedings, 1997, 1212-1215.

[53] Mission needs Statement for the Joint Tactical Radio, 1997 - 2012

[54] P. Mackenzie, An architecture for the development of software Radios on General Purpose Processors, Proceedings of the IRISH Signals \& system conference, 2002, 275-280.

[55] V.G. Bose, A software Driven Approach to SDR design, COTS Journal, 2004.

[56] www.gnu.org

[57] G. Youngblood, a Software Defined Radio for the Masses Part 1, QEX: A forum for Communication Experiments, 2002.

[58] C. C. Lin, Design and Implementation of Software Framework for software defined Radio System, Proceedings of the $56^{\text {th }}$ IEEE Vehicular Technology conference, 2002, 2395-2399.

[59] G. J. Minden, Agile Radio system and National Radio Networking Research Testbed, Sensornet Architecture Forum, Lawrence, KS, USA, 2003.

[60] www.calradio.calit2.net

[61] P. Murthy, Design of WARP: A Flexible Wireless Open Access Research Platform, Proceedings of $14^{\text {th }}$ European Signal Processing Conference, 2006.

[62] www.openhpsdr.org

[63] J. A. Depries, A Practical approach to rapid prototyping of SCA waveforms, Master diss., Virginia Polytechnic Institute and State University, Blacksburg, Virginia, USA, 2006.

[64] Lyrtech SFF SDR Development Platform Technical Specs, Technical report, 2007

[65] T. W. Rondeau, Application of Artificial Intelligence to Wireless communication, Doctoral diss., Virginia Polytechnic Institute and State University, Blacksburg, Virginia, USA, 2007
[66] G. Kim, Early Results on HYDRA: A flexible MAC/PHY Multihop Testbed, Proceedings of $65^{\text {th }}$ IEEE Vehicular Technology Conference, 2007.

[67] I. G. Miguelez, A software framework for software radio, Master diss., Universidad Politecnica de cataluna, Barcelona, 2008.

[68] K. Tan, Sora: High Performance Software Radio using General Purpose Multi Core processors, P6th USENIX Symposium on Networked System Design and Implementation, 2009.

[69] Native Interference between MATLAB/Simulink and USRP.

[70] www.mathworks.com

[71] gnsssdr.org 